\title{
Instabilities of vortex rings generated by surface-tension gradients between co-axial disks ${ }^{\text {th }}$
}

\author{
Qi-Sheng Chen *, Yan-Ni Jiang \\ Key Laboratory of Microgravity (National Microgravity laboratory), Institute of Mechanics, Chinese Academy of Sciences, 15 Bei Si Huan Xi Road, Beijing 100190, China
}

\section{A R T I C L E I N F O}

Available online 11 September 2012

\section{Keywords:}

Liquid bridge

Thermocapillary flow

Surface-tension gradients

Vortex rings

\begin{abstract}
A B S T R A C T
Instabilities of vortex rings generated by surface-tension gradients between co-axial disks with Prandtl numbers of 0.001 and 0.01 are investigated by using the linear stability analysis (LSA) method. The continuity-vortex-energy equations are used as the perturbation equations for stability analysis and discretized using a Chebyshevcollocation method. The critical Reynolds numbers and the angular wavenumbers of the unstable mode are obtained for vortex rings with a range of aspect ratios between 0.05 and 1.2. From stability analyses, it is found that the product of the critical model and the aspect ratio approaches a constant when the aspect ratio decreases. The critical mode is $m=16$ when the aspect ratio is 0.05 . Analyses also indicate that the vortex rings must be of certain energy for the perturbations of flow to grow in amplitude. The viscosity of fluid can dampen perturbations if the magnitude of the stream function of the basic flow is below the critical value. The vortex rings generated by surface-tension gradients become unstable when the magnitude of the stream function is above the critical value.
\end{abstract}

(c) 2012 Elsevier Ltd. All rights reserved.

\section{Introduction}

The study of vortex rings has been pursued by many researchers for many years. Widnall and Sullivan [1] investigated theoretically and experimentally the stability of vortex rings. The effect of the vorticity distribution within the finite vortex core on the self-induced motion of each element of the vortex filament was studied. Widnall and Tsai [2] investigated theoretically the instability of a thin vortex ring with a core of constant vorticity in an ideal fluid to short azimuthal bending waves. Vortex rings can also be found in applications such as floatingzone and Czochralski crystal-growth processes [3]. Crystal growth conducted under microgravity conditions has had a profound impact on improving the understanding of melt crystal growth processes [4]. Campbell et al. [5] studied the macrosegregation in the crystals grown with the floating-zone technique. However, the formation mechanism of dopant striations during zone refining of materials hasn't been explained quantitatively. For small Prandtl numbers $(\operatorname{Pr} \ll 1)$ the first instability of the axisymmetric flow is a stationary bifurcation. Levenstam and Amberg [6] simulated the transition from the axisymmetric thermocapillary flow to a steady non-axisymmetric flow with an azimuthal wavenumber of 2 for a half-zone with Prandtl number of 0.01 and aspect ratio of 0.5 . Wanschura et al. [7] simulated the instability of the axisymmetric steady thermocapillary flow in a cylindrical liquid bridge by using a mixed Chebyshev-finite difference method. Chen et al. [8] calculated the critical Marangoni numbers as a function of liquid volume for liquid bridges with Prandtl numbers of $0.001,0.01$, and 0.1 by using the linear stability analysis (LSA) method. Lappa et al. [9] simulated the three-dimensional instability of the liquid bridge with $\operatorname{Pr}=0.01$. Nienhüser and Kuhlmann [10] investigated the stability of thermocapillary flows in non-cylindrical liquid bridges. The hydrostatic shape of the bridge is the most important effect on the critical point in a gravity field.

In spite of the relatively simple geometry of the half-zone, factors such as liquid volume, aspect ratio, surface deformation and the physical properties of the liquid make comparisons between experiments complicated. It is ideal to find a model that can explain the instabilities of vortex rings in liquid bridge or Czochralski configurations. The study of stabilities of vortex rings by surface-tension gradients will improve the understanding of crystal growth processes, and explain the instability mechanism of vortex rings. We consider here liquids with Prandtl numbers of 0.001 and 0.01 to reduce the effect of perturbations of surface-tension gradients.

\section{Problem formulation}

The floating half-zone model consists of a liquid bridge held between two solid planar disks of diameter $D$, which are separated by a distance $L$ (Fig. 1). The upper disk usually has a temperature, $T_{0}+\Delta T$, higher than the temperature at the lower disk, $T_{0}$, to reduce the buoyancy effect. Typical dimensionless parameters such as aspect ratio, Reynolds number, Marangoni number, Prandtl numbers and Biot number are defined as,

$A=\frac{L}{2 R_{0}}, \operatorname{Re}=\frac{U_{0} R_{0}}{\nu}, \operatorname{Ma}=\frac{U_{0} R_{0}}{\alpha}, \operatorname{Pr}=\frac{\nu}{\alpha}, \mathrm{Bi}=\frac{h R_{0}}{k}$,

\footnotetext{
Communicated by P. Cheng and W.Q. Tao.

* Corresponding author.

E-mail address: qschen@imech.ac.cn (Q.-S. Chen).
} 


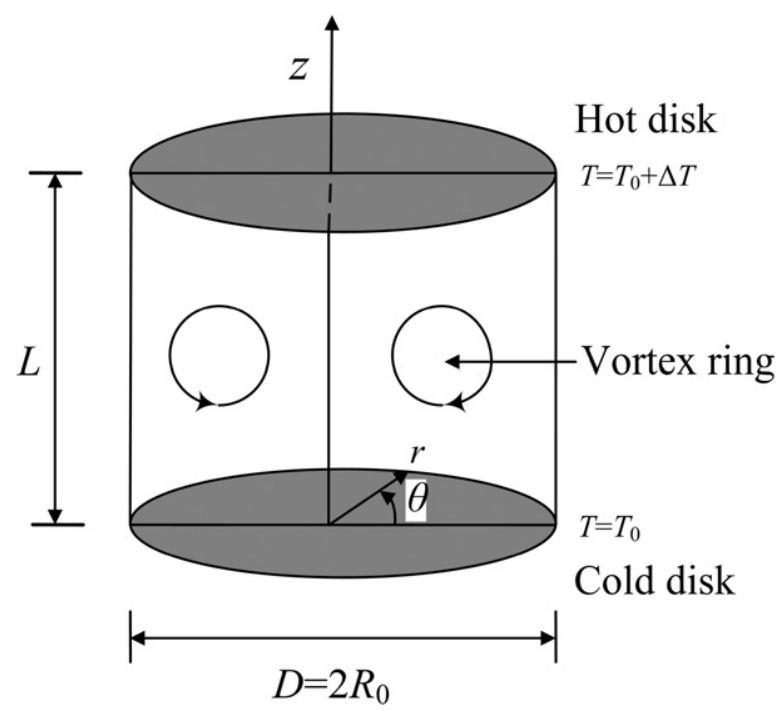

Fig. 1. Schematic of a vortex ring generated by surface-tension gradients in a floating half-zone between two planar disks.

where the reference velocity is $U_{0}=\left|\sigma_{T}^{\prime}\right| \Delta T / \rho \nu$, and $R_{0}, \rho, \nu, \alpha, k, h$, and $\sigma_{T}^{\prime}$ denote disk radius, liquid density, kinematic viscosity, thermal diffusivity, thermal conductivity, heat transfer coefficient, surfacetension derivative with respect to temperature, respectively.

For calculation of axisymmetric basic state of the surface-tension driven convections in floating half-zone between disks, the liquid is considered as incompressible. A steady axisymmetric basic state, $\mathbf{u}_{0}=\left(u_{0}, 0, w_{0}\right), p_{0}$ and $T_{0}$, is obtained by the spectral-collocation method [11,12]. To derive the dimensionless control equations, the length, time, velocity and pressure scales are set as $R_{0}, R_{0}^{2} / \nu, \nu / R_{0}$, and $\rho_{0} v^{2} / R_{0}{ }^{2}$, respectively. The equations are as follows,

$\nabla \cdot \mathbf{u}_{0}=0$

$\frac{\partial \mathbf{u}_{0}}{\partial t}+\mathbf{u}_{0} \cdot \nabla \mathbf{u}_{0}=-\nabla p_{0}+\frac{1}{\operatorname{Re}} \nabla^{2} \mathbf{u}_{0}$,

$\frac{\partial T_{0}}{\partial t}+\mathbf{u}_{0} \cdot \nabla T_{0}=\frac{1}{\operatorname{RePr}} \nabla^{2} T_{0}$.

The boundary conditions at $z=0$ are

$u_{0}=w_{0}=0$, and $T_{0}=0$,

while the boundary conditions at $z=2 A$ are

$u_{0}=w_{0}=0$, and $T_{0}=1$.

The boundary conditions at the axis are chosen as

$u_{0}=0, \frac{\partial w_{0}}{\partial r}=0$, and $\frac{\partial T_{0}}{\partial r}=0$.

The boundary conditions at the free surface are

$\mathbf{u}_{0} \cdot \mathbf{n}=0, \mathbf{t} \cdot \mathbf{S}_{0} \cdot \mathbf{n}=-\frac{\mathrm{Ma}}{\operatorname{RePr}} \boldsymbol{t} \cdot \nabla T_{0}$, and $\mathbf{n} \cdot \nabla T_{0}=-\mathrm{Bi} T_{0}$,

where $\mathbf{n}$ denotes the normal unit vector at the free surface, $\mathbf{t}$ denotes the tangential unit vectors in the vertical cross-section, and $\mathbf{S}_{0}$ denotes the rate-of-strain tensor. The viscous tangential stress is balanced by the surface-tension gradient at the free surface, and the minus sign on the right side of the equations arrives since liquids usually have a negative coefficient of the surface tension with regard to temperature. The Biot number is set as $\mathrm{Bi}=0$ in the present calculations.

In linear stability analysis of surface-tension driven convections in the floating half-zone, the small-amplitude fluctuations of the velocities $\mathbf{u}=(u, v, w)$, pressure $p$ and temperature $T$ are imposed on the basic state. The dimensionless governing equations for the perturbation quantities under the microgravity conditions can be obtained as in [8]. After applying the curl operator to the momentum equation to eliminate the pressure term, we can obtain the vorticity equations. For the stability analysis, we use the continuity equation, the vorticity equations in $r$ and $z$ directions, and the energy equation for solving the eigenvalue problem. The continuity equation is included in the perturbation equations to ensure that the continuity equation is satisfied at all grid points. The perturbation quantities $(u, v, w, p, T)$ can be expanded as a sum of the spectral terms,

$$
\left(\begin{array}{c}
u \\
v \\
w \\
p \\
T
\end{array}\right)=\sum_{m} e^{\sigma t+i m \theta}\left(\begin{array}{l}
\tilde{u}_{m}(r, z) \\
i m \tilde{v}_{\mathrm{m}}(r, z) \\
\tilde{w}_{m}(r, z) \\
\tilde{p}_{m}(r, z) \\
\tilde{T}_{m}(r, z)
\end{array}\right)+\text { c.c. }
$$

where $\sigma=\sigma_{r}+i \sigma_{i}, \sigma_{r}$ and $\sigma_{i}$ are the amplification rate and frequency of small perturbation, respectively, $m$ denotes the azimuthal wavenumber, $i$ denotes the complex unit $\sqrt{-1}$, and c.c. denotes the complex conjugate.

The Chebyshev-collocation method is used to calculate the basic states of flow and temperature with $49 \times 73$ Chebyshev polynomials in $r$ and $z$ directions, respectively. The collocation method is also used to solve the discretized equations for the eigenvalue problem. We use $33 \times 33$ Chebyshev polynomials in $r$ and $z$ directions for discretizing the perturbation equations. The eigenvalues and eigenfunctions are then obtained by using the Q-R method. Since four perturbation equations are used, the complex matrix for the generalized eigenvalue problem has a size of $4356 \times 4356$.

\section{Results}

\subsection{Choosing of azimuthal wavenumbers}

In studying the instability of the thin vortex ring of constant vorticity, Widnall and Tsai [2] found that the wavelength is proportional to the core radius so that $k a=\kappa$ where $k$ is the angular wavenumber (defined as $k=2 \pi / \lambda, \lambda$ is the wavelength). Stability analysis showed that by assuming the perturbed waves do not rotate, the line vortex is always unstable for at least the lowest two critical dimensionless wavenumbers $\kappa=2.5$ and 4.35 ( $\kappa$ is the wavenumber non-dimensionalized by the core radius $a$ in the stability analysis). This implies that the wavelength of the most unstable wave on a vortex ring is a fixed number of vortex core radii, independent of vortex-ring radius. In dimensionless form, the relation is

$m \varepsilon=\kappa$,

where $\varepsilon=a / R_{\text {ring }}$ is the aspect ratio of vortex ring, $m=2 \pi R_{\text {ring }} / \lambda$ is the dimensionless azimuthal wavenumber, and $R_{\text {ring }}$ is the radius of the vortex ring.

The computed critical values by LSA are listed in Tables 1 and 2 . It appears that the computed azimuthal wavenumber increases $(1 \leq m \leq 16)$ as the aspect ratio $A$ decreases from 1.2 to 0.05 . The radii of the roll axis of the basic flow, $R_{\text {ring, }}$, are also listed. The ratio of the vortex-ring radius and the disk radius increases as the aspect ratio decreases. The centrifugal force pushes the vortex ring structure to the outer region of the liquid bridge when the aspect ratio is small, and the cross-section of the vortex core is not circular. In making the analogy between a vortex ring and the liquid bridge, Levenstam and 
Table 1

Critical values for surface-tension driven flows with $\operatorname{Pr}=0.001$.

\begin{tabular}{lcccc}
\hline$A$ & The critical mode & The critical Reynolds number & $m A$ & $R_{\text {ring }} / \mathrm{R}$ \\
\hline 0.05 & 16 & 48,307 & 0.8 & 0.962 \\
0.1 & 9 & 15,020 & 0.9 & 0.932 \\
0.2 & 4 & 6093 & 0.8 & 0.876 \\
0.3 & 3 & 3435 & 0.9 & 0.832 \\
0.4 & 2 & 2566 & 0.8 & 0.802 \\
0.5 & 2 & 1871 & 1 & 0.777 \\
0.6 & 2 & 1402 & 1.2 & 0.750 \\
0.8 & 2 & 1070 & 1.6 & 0.732 \\
1 & 1 & 731 & 1 & 0.728 \\
1.1 & 1 & 610 & 1.1 & 0.726 \\
1.2 & 1 & 550 & 1.2 & 0.723 \\
\hline
\end{tabular}

Amberg [6] assumed that the vortex core diameter to be the height of the liquid bridge. From Tables 1 and 2, it appears that the product of the azimuthal wavenumber of the unstable mode and the aspect ratio approaches a constant,

$m A \approx 0.8$,

when $0.05 \leq A \leq 0.4$.

There is no theoretic data on the instabilities of Marangoni flows in small-aspect-ratio liquid bridges. The only available experimental data on critical wavenumbers are by Preisser et al. [13] who studied the transition of a thermocapillary convection to an oscillatory convection in liquid columns with $\operatorname{Pr}=7$ and found that $m A \approx 1.1$ when $0.2 \leq A \leq 0.5$. It should be noted that at $\operatorname{Pr}=7$, amplification of disturbances in surface-tension gradient is significant in the bifurcated solutions. However, the hydrodynamic effect is still dominant for large-Prandtl-number surface-tension driven flows when the aspect ratio is small.

The product of $m A$ obtained by the LSA method is plotted in Fig. 2 along with the experimental data [1]. The aspect ratios $\varepsilon=a / R_{\text {ring }}$ in [1] have been multiplied by a factor of 0.75 to suit the current definition of the aspect ratio of liquid bridge assuming that $R_{\text {ring }}$ in [1] is about 0.75 times radius of the periphery where the tangential velocity peaks. The experiments in Ref. [1] dealt with vortex rings in the air, and $m A$ decreases when aspect ratio decreases (Fig. 2). The $m A$ obtained for surface-tension driven flows is generally lower than the $m A$ obtained for the vortex rings in the air. This may be due to the fact that vortex rings by surface-tension gradients are bounded by solid walls and vortex rings in the air are unbounded. It appears that the instabilities for vortex rings induced by surfacetension gradients and generated in the air are both of hydrodynamic in nature. Since the melts are generally opaque it is difficult to obtain experimental data for instabilities of small-Prandtl-number surface-tension driven flows.

Table 2

Critical values for surface-tension driven flows with $\operatorname{Pr}=0.01$.

\begin{tabular}{llcll}
\hline$A$ & The critical mode & The critical Reynolds number & $m A$ & $R_{\text {ring }} / \mathrm{R}$ \\
\hline 0.05 & 16 & 51,928 & 0.8 & 0.962 \\
0.1 & 8 & 17,262 & 0.8 & 0.933 \\
0.2 & 4 & 6620 & 0.8 & 0.877 \\
0.3 & 3 & 3666 & 0.9 & 0.832 \\
0.4 & 2 & 2687 & 0.8 & 0.802 \\
0.5 & 2 & 1972 & 1 & 0.774 \\
0.6 & 2 & 1500 & 1.2 & 0.751 \\
0.8 & 2 & 1186 & 1.6 & 0.726 \\
1 & 1 & 776 & 1 & 0.724 \\
1.1 & 1 & 653 & 1.1 & 0.723 \\
1.2 & 1 & 596 & 1.2 & 0.723 \\
\hline
\end{tabular}

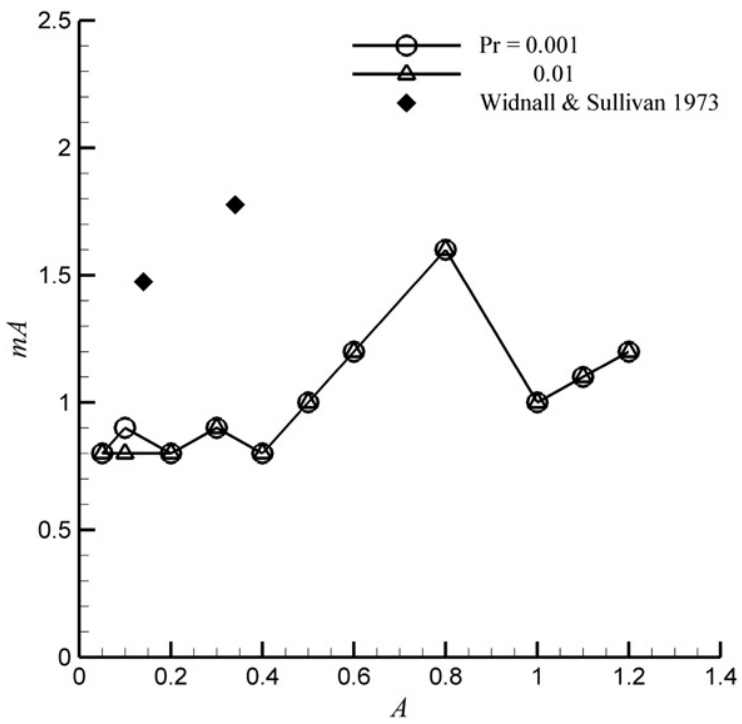

Fig. 2. Dependence of $m A$ on the aspect ratio for the unstable modes of the surface-tension driven flows. Symbol circle, $\operatorname{Pr}=0.001$; symbol delta, $\operatorname{Pr}=0.01$; filled diamond, experimental data [1].

\subsection{Features of the unstable mode}

Here the features of the unstable mode of the vortex ring with $m=9$ are presented. The contours of the amplification rate $\sigma_{\mathrm{r}} \operatorname{Re}$ are plotted in Fig. 3 for $\operatorname{Pr}=0.001$ and $A=0.1$. From the neutral curve $\sigma_{\mathrm{r}} \operatorname{Re}=0$, we can estimate that the critical mode is $m=9$. The amplification rate as a function of Reynolds number for $A=0.1$ and $m=9$ can be linearly fitted as, $\sigma_{r} \operatorname{Re}=0.028 *(\operatorname{Re}-15,020)$. In the analysis by Widnall and Tsai [2], the linear instability of a thin vortex ring was performed based on the assumption of inviscid flow, and the amplification rate was found to be proportional to the total circulation. The present calculations indicate that vortex rings must be of certain energy for the perturbation of flow on the vortex to occur and grow in amplitude [1]. Here we use the stream function to represent the convection strength. If the magnitude of the stream function of the convections is smaller than the critical value, the viscosity of fluid can dampen any perturbations. When the magnitude of the stream function is above the critical value the vortex rings generated by surface-tension gradients become unstable.

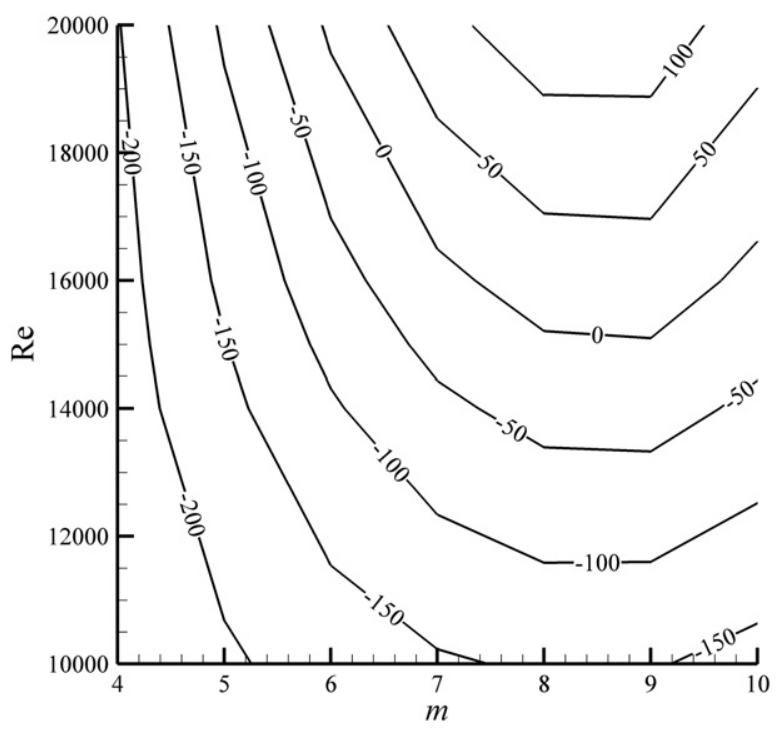

Fig. 3. The contours of the amplification rate $\sigma_{r}$ Re for $\operatorname{Pr}=0.001$ and $A=0.1$. 
a)

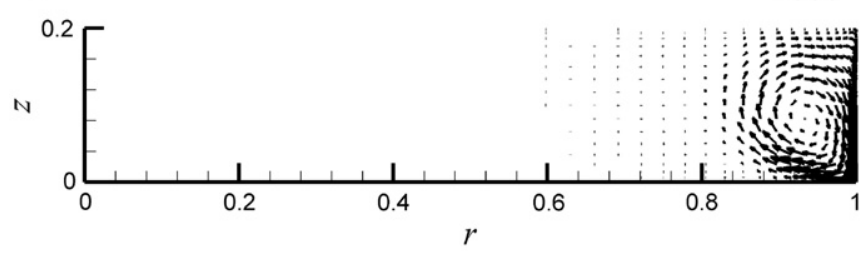

b)

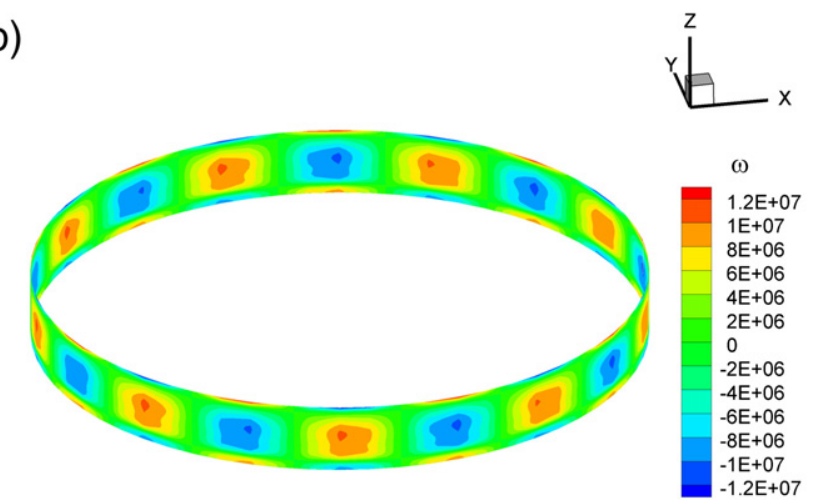

Fig. 4. (a) Basic flow and (b) distribution of the perturbed vorticity in the azimuthal direction at $r=0.88$ when $\operatorname{Pr}=0.001, A=0.1$ and $\operatorname{Re}=16,000$.

The typical basic flow and the distribution of the perturbed vorticity in the azimuthal direction $\omega=\frac{\partial u}{\partial z}-\frac{\partial w}{\partial r}$, are plotted in Fig. 4(a) and (b), respectively, when $\operatorname{Pr}=0.001, A=0.1$, and $\operatorname{Re}=16,000$. The basic flow is confined in the region near the free surface of the liquid column, and a vortex ring is generated. The corresponding eigenvalue for the basic state is $\left(\sigma_{\mathrm{r}}+i \sigma_{\mathrm{i}}\right) \operatorname{Re}=26.78+i 0$. The perturbation calculated by LSA does not rotate since $\sigma_{\mathrm{i}}$ is zero. The disturbance solution is normalized using the maximum temperature of the perturbation. The perturbation flow in the unstable mode contains nine waves around the perimeter at $r=0.88$. Each perturbed wave generates strong positive and negative vorticities in the azimuthal direction. The positive and negative perturbed vorticities will result in sinusoidal displacements of the centerline of vortex ring in both the radial and vertical directions.

\section{Summary}

Instabilities of vortex rings generated by surface-tension gradients between co-axial disks with Prandtl numbers of 0.001 and 0.01 are investigated by using the linear stability analysis method. From the stability analysis, it appears that the product of the angular wavenumber of the unstable mode and the aspect ratio approaches a constant when the aspect ratio decreases. That means that the wavelength of the unstable mode is proportional to the radius of the vortex core, and the instability is a local behavior of vortex when the aspect ratio is small. By considering the viscosity of fluid, the stability analyses indicate that the vortex rings must be of certain energy for the perturbation of flow to grow in amplitude. The vortex rings driven by surface-tension gradients are unstable when the magnitude of the stream function is above a critical value. This suggests that the viscosity of fluid can dampen perturbations if the magnitude of the stream function of the basic flow is below the critical value. Experiments will be performed in the future to study the features of vortex rings in both shallow liquid bridge and Czochralski configurations.

\section{Acknowledgments}

The work was supported by the National Natural Science Foundation of China under grant nos. 10972226 and 10621202.

\section{References}

[1] S.E. Widnall, J.P. Sullivan, On the stability of vortex rings, Proceedings of the Royal Society of London. Series A 332 (1973) 335-353.

[2] S.E. Widnall, C.-Y. Tsai, The instability of the thin vortex ring of constant vorticity, Philosophical Transactions of the Royal Society A 287 (1977) 273-305.

[3] M.F. Schatz, G.P. Neitzel, Experiments on thermocapillary instabilities, Annual Review of Fluid Mechanics 33 (2001) 93-127.

[4] J.J. Derby, Y.I. Kwon, A. Pandy, P. Sonda, A. Yeckel, T. Jung, G. Müller, Developing quantitative, multiscale models for microgravity crystal growth, Annals of the New York Academy of Sciences 1077 (2006) 124-145.

[5] T.A. Campbell, M. Schweizer, P. Dold, A. Cröll, K.W. Benz, Float zone growth and characterization of $\mathrm{Ge}_{1-\mathrm{x}} \mathrm{Si}_{\mathrm{x}}(\mathrm{x} \leq 10$ at\%) single crystals, Journal of Crystal Growth 226 (2001) 231-239.

[6] M. Levenstam, G. Amberg, Hydrodynamical instabilities of thermocapillary flow in a half-zone, Journal of Fluid Mechanics 297 (1995) 357-372.

[7] M. Wanschura, V.M. Shevtsova, H.C. Kuhlmann, H.J. Rath, Convective instability mechanisms in thermocapillary liquid bridges, Physics of Fluids 5 (1995) 912-925.

[8] Q.-S. Chen, W.-R. Hu, V. Prasad, Effect of liquid bridge volume on the instability in small-Prandtl-number half zone, Journal of Crystal Growth 203 (1999) 261-268.

[9] M. Lappa, R. Savino, R. Monti, Three-dimensional numerical simulation of Marangoni instabilities in non-cylindrical liquid bridges in microgravity, International Journal of Heat and Mass Transfer 44 (2001) 1983-2003.

[10] C. Nienhüser, H.C. Kuhlmann, Stability of thermocapillary flows in non-cylindrical liquid bridges, Journal of Fluid Mechanics 458 (2002) 35-73.

[11] S.A. Orszag, L.C. Kells, Transition to turbulence in plane Poiseuille and plane Couette flow, Journal of Fluid Mechanics 96 (1980) 159-205.

[12] Q.S. Chen, W.R. Hu, Numerical investigation on a simulation model of floating zone convection, International Journal of Heat and Mass Transfer 40 (1997) 757-763.

[13] F. Preisser, D. Schwabe, A. Scharmann, Steady and oscillatory thermocapillary convection in liquid columns with free cylindrical surface, Journal of Fluid Mechanics 126 (1983) 545-567. 